Copyright (C1996, American Institute of Aeronautics and Astronautics, Inc.

AIAA Meeting Papers on Disc, September 1996

A9641225, AIAA Paper 96-4250

\title{
Electrodynamic tethers for reboost of the International Space Station and spacecraft propulsion
}

\author{
Les Johnson \\ NASA, Marshall Space Flight Center, Huntsville, AL \\ Joe Carroll \\ Tether Applications Co., Chula Vista, CA \\ Robert D. Estes \\ Smithsonian Astrophysical Observatory, Cambridge, $M A$ \\ Enrico Lorenzini \\ Smithsonian Astrophysical Observatory, Cambridge, $M A$ \\ Brian Gilchrist \\ Michigan Univ., Ann Arbor \\ Manuel Martinez-Sanchez \\ MIT, Cambridge, $M A$ \\ Juan Sanmartin \\ Madrid, Polytechnic Univ., Spain \\ Irwin Vas \\ Boeing Defense \& Space Group, Huntsville, AL
}

AIAA, Space Programs and Technologies Conference, Huntsville, AL, Sept. 24-26, 1996

The International Space Station (ISS) will require periodic reboost due to atmospheric aerodynamic drag. This is nominally achieved through the use of thruster firings by the attached Progress $M$ spacecraft. Many Progress flights to the ISS are required annually. Electrodynamic tethers provide an attractive alternative in that they can provide periodic reboost or continuous drag cancellation using no consumables, propellant, nor conventional propulsion elements. The system could also serve as an emergency backup reboost system used only in the event resupply and reboost are delayed for some reason. The system also has direct application to spacecraft and upper stage propulsion. Electrodynamic tethers have been demonstrated in space previously with the Plasma Motor Generator (PMG) experiment and the Tethered Satellite System (TSS-IR). The advanced electrodynamic tether proposed for this application has significant advantages over previous systems in that higher thrust is achievable with significantly shorter tethers and without the need for an active current collection device, hence making the system simpler and much less expensive. (Author) 


\title{
ELECTRODYNAMIC TETHERS FOR REBOOST OF THE INTERNATIONAL SPACE STATION AND SPACECRAFT PROPULSION
}

\author{
Les Johnson, NASA Marshall Space Flight Center \\ Huntsville, Alabama \\ Joe Carroll, Tether Applications Company \\ Chula Vista, California \\ Robert D. Estes \& Enrico Lorenzini, Smithsonian Astrophysical Observatory \\ Cambridge, Massachusetts \\ Brian Gilchrist, The University of Michigan \\ Ann Arbor, Michigan \\ Manuel Martinez-Sanchez, Massachusetts Institute of Technology \\ Cambridge, Massachusetts \\ Juan Sanmartín, Polytechnic University of Madrid \\ Madrid, Spain \\ Irwin Vas, Boeing Space and Defense Company \\ Huntsville, Alabama
}

\begin{abstract}
$\underline{\text { Abstract }}$
The International Space Station (ISS) will require periodic reboost due to atmospheric aerodynamic drag. This is nominally achieved through the use of thruster firings by the attached Progress $M$ spacecraft. Many Progress flights to the ISS are required annually. Electrodynamic tethers provide an attractive alternative in that they can provide periodic reboost or continuous drag cancellation using no consumables, propellant, nor conventional propulsion elements. The system could also serve as an emergency backup reboost system used only in the event resupply and reboost are delayed for some reason. The system also has direct application to spacecraft and upper stage propulsion.
\end{abstract}

Electrodynamic tethers have been demonstrated in space previously with the Plasma Motor Generator (PMG) experiment and the Tethered Satellite System (TSS-1R). The advanced electrodynamic tether proposed for this application has significant advantages over previous systems in that higher thrust is achievable with significantly shorter tethers and without the need for an active current collection device, hence making the system simpler and much less expensive.

\section{Propellantless Reboost for the ISS: An Electrodynamic Tether Thruster}

The need for an altemative to chemical thruster reboost of the $I S S$ has become increasingly apparent as the station nears completion. We propose a system to utilize ISS electrical power to generate thrust by means of a new type of electrodynamic tether attached to the station (Fig. 1). A flexible system could be developed to generate an average thrust of 0.5 to $0.8 \mathrm{~N}$ for 5 to 10 $\mathrm{kW}$ of electrical power. By comparison, aerodynamic drag on $I S S$ is expected to average from 0.3 to $1.1 \mathrm{~N}$ (depending upon the year).

The proposed system uses a tether with a kilometers-long uninsulated (bare) segment capable of collecting currents greater than $10 \mathrm{~A}$ from the ionosphere. The new design exhibits a remarkable insensitivity to electron density variations, allowing it

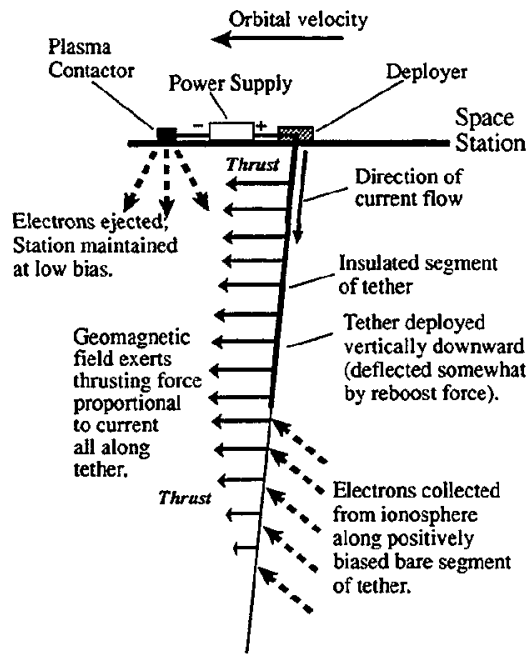

Fig. 1. An electrodynamic tether reboost system for the ISS.

1

American Institute of Aeronautics and Astronautics 
to operate efficiently even at night. A relatively short and light tether $(10 \mathrm{~km}$ or less, $200 \mathrm{~kg})$ is required, thus minimizing the impact on the ISS (center of mass shift less than $5 \mathrm{~m}$ ).

The groundwork has been laid for a tether reboost system. NASA has developed tether technology for space applications over the past several years. Important milestones include retrieval of a tether in space (TSS-1, 1992), successful deployment of a $20-\mathrm{km}$-long tether in space (SEDS-1, 1993), and operation of an electrodynamic tether with tether current driven in both directions-power and thrust modes (PMG, 1993).

Most important for the reboost application, TSS-1R recently drew currents on the order of $1 \mathrm{~A}$, which corresponded to a magnetic force on the Shuttle-tether system of around 0.4 Newtons (though as a drag rather than a thrust due to the direction of current flow in the tether). To summarize: the predicted behavior of tethered systems, dynamically and electrodynamically, has been confirmed in space.

\section{High Tether Currents for ISS Reboost}

Basic Principles and Technical Challenges. An electrodynamic tether can work as a thruster because a magnetic field exerts a force on a current-carrying wire. This force is perpendicular to the wire and to the field vector. If the current flows downward through a tether connected to the ISS, the force exerted by the geomagnetic field on the system has a component that accelerates the station along the direction in which it is already moving.

An orbiting system, by virtue of its motion through the Earth's magnetic field, experiences an electric field $(\mathbf{v} \times \mathbf{B})$ perpendicular to its direction of motion and to the geomagnetic field vector. For an eastward-moving system, such as the ISS, the field is such that the electrical potential decreases with increasing altitude (at a rate of around $100 \mathrm{~V} / \mathrm{km}$ for $I S S$ orbit). In order to drive a current down the tether, it is necessary to overcome this induced e.m.f.

Thus, our reboost system requires a power supply and may be considered a type of electrical thruster. Calculations for the preliminary design indicate an average thrust of $0.5 \mathrm{~N}$ from $5 \mathrm{~kW}$ and $0.8 \mathrm{~N}$ from $10 \mathrm{~kW}$. The tether is $10-\mathrm{km}$ long with a mass less than $200 \mathrm{~kg}$. Our assumption is that there will be times during the day when surplus power from the solar panels could be utilized for this thruster power and that, depending upon the need and power reserves, night operation on battery power may also be possible.

We propose to utilize a plasma contactor on the ISS to eject electrons; thus, we are driven to deploy the tether vertically downward for a reboost application. Thanks to the power supply, which is placed in series between the plasma contactor and the upper end of the tether (see Fig. 1), the upper end of the tether is at a higher electrical potential than the plasma for some distance below it. This distance may be greater than the tether length if the applied voltage exceeds the motional e.m.f. Now the ionospheric electrons below the station would "like" to get to the higher potential at the upper end of the tether. If the electrons can make contact with the tether, they will travel up it, giving a current flow in the correct direction for reboost.

The way in which the charge exchange between tether and plasma takes place depends upon the specifics of the system, and it is this aspect (specifically the electron collection, which is the difficult part) that we have focused on in designing a system capable of producing sufficient thrust with a reasonably short tether for the ISS.

The magnitude of the thrust force is dependent upon the motional e.m.f. (between the two ends of the tether), the average current in the tether and the orbital speed. Thus, the product of the tether length and average tether current determines the thrust for given orbital/magnetic conditions. Generally speaking, a shorter tether will have a smaller impact on the ISS environment; so a combination of high current with short tether length is the goal.

ISS reboost (thrust forces of order $1 \mathrm{~N}$ ) with a tether no longer than $10 \mathrm{~km}$ requires tether currents of order $10 \mathrm{~A}$. The critical issue is how to draw ionospheric electrons at that rate. The standard tether carries insulation along its entire length, exchanging current with the ionosphere only at the ends: TSS-1R carried a passive metallic sphere as anode; PMG carried an active (plasma-ejecting) contactor.

Current collected to a passive, biased sphere in a magnetized plasma calculated by the standard ParkerMurphy (PM) model (taking into account magnetic effects, which are dominant) grows as the square-root of the bias voltage, an important fact for fixed-area collectors.

A preliminary analysis of the measured TSS-1R currents indicates that they were typically greater than the PM model predictions (using values of the electron density and temperature estimated from ionospheric models and a satellite voltage calculated with some uncertainty). The TSS-1R data do not, however, appear to point to a dependence of current on voltage greatly different from that of PM for higher voltages. Even though, for example, a TSS-1R current of $0.5 \mathrm{~A}$ at $350 \mathrm{~V}$ bias may surpass PM model estimates, it could still imply a voltage of roughly $35 \mathrm{kV}$ to reach $5 \mathrm{~A}$ for 
the same plasma parameters (which would require over $175 \mathrm{~kW}$ for a thrust of $0.7 \mathrm{~N}$ with a $10-\mathrm{km}$-long tether!).

Active anodes (plasma contactors) have been developed in an attempt to solve both space-charge shielding and magnetic guiding effects by creating a self-regulating plasma cloud to provide quasineutrality and by emitting ions to counterstream attracted electrons and produce fluctuations that scatter those electrons off magnetic field lines. The only tether experiment to use an active anode so far was the PMG, which reached $0.3 \mathrm{~A}$ in flight under a $130-\mathrm{V}$ bias and the best ionospheric conditions. Unfortunately, there is no way to scale the results to high currents. The discouraging fact was that collected current decreased sharply with the ambient electron density at night.

Fortunately, there is another tether design optionthe bare tether. ${ }^{1}$

The Bare-Tether Breakthrough. The bare-tether design represents a breakthrough that makes short-tether electrodynamic reboost with moderate power requirements for the $I S S$ practical. To work on the $I S S$, a reboost system must not only be capable of delivering adequate thrust (preferably night and day); it must do so with small impact on the ISS environment while requiring minimal accommodation by the baseline ISS systems. It should also be simple to operate and maintain, and it must be competitive in terms of its use of resources for the benefits it provides.

Our proposed design uses the tether itself, left uninsulated over the lower portion, to function as its own very efficient anode. The tether is biased positively with respect to the plasma along some or all of its length. The positively biased, uninsulated part of the tether then collects electrons from the plasma.

The following features argue in favor of the baretether concept.

(1) The small cross-sectional dimension of the tether makes it a much more effective collector of electrons (per unit area) from the space plasma than as a large sphere (such as the TSS-1R satellite) at equal bias. This is because the small cross dimension of the tether allows its current collection to take place in the orbital-motion-limited regime, which gives the highest possible current density.

(2) The large current-collection area is distributed along the tether itself, eliminating the need for a large, massive and/or high-drag sphere or a resource-using plasma contactor at the upper end of the tether. This substantially reduces the center of gravity shift in both cases and reduces the cost and complexity in the case of the active contactor.

(3) The system is self-adjusting to changes in electron density. This is accomplished by a natural expansion of the portion of the tether that is biased positively relative to the ionosphere whenever the density drops (Fig. 2) as described by Sanmartin, et al.

Features (1) and (2) combine to provide an ability to collect large currents with modest input power levels. We present below a candidate system that can produce average thrusts of 0.5 to $0.8 \mathrm{~N}$, for input power of 5 to $10 \mathrm{~kW}$.

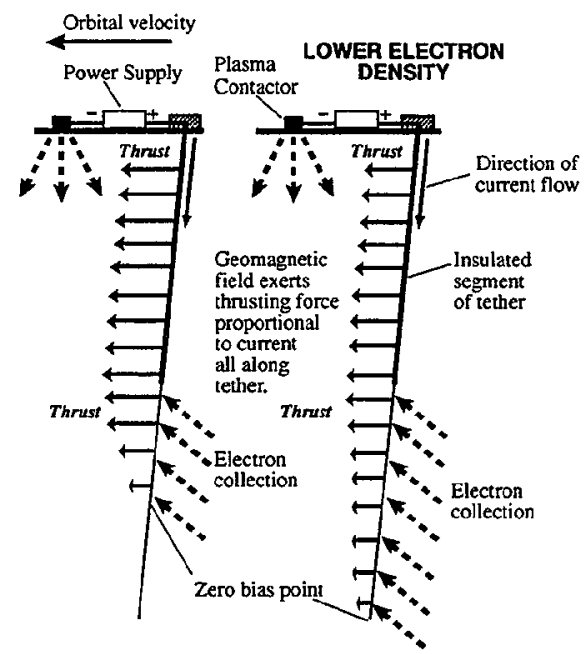

Fig. 2. A bare-tether thruster designed to adjust to lower electron density (as at night). A shift in the zero point of bias further down the tether increases the collecting surface and maintains a nearly steady thrust for constant input power and induced e.m.f.

The Bare-Tether Solution: Theoretical and Experimental Basis. With an eye to our tether design, let us now review the current-collecting properties of a long, thin cylinder, for which there are well-established theoretical and experimental results. Charged-particle collection is governed by the stronger gradients associated with the smaller dimensions and is thus a two-dimensional (2-D) process, the length being irrelevant to the density of current collected.

For a radius that is small compared to both Debye length and gyroradius, there are neither space-charge nor magnetic-guiding effects, and we are in the orbitalmotion-limited regime of standard Langmuir theorycalled OML in what follows. In the OML regime, the current takes the largest possible value for the given 
geometry and bias. Better still, it turns out that in cylindrical geometry the OML regime holds for radiusto-Debye length ratios even of order unity. ${ }^{2}$ Hence, a cylinder of 5-mm radius (about one Debye length and small compared with gyroradius) works in the OML regime.

For a cylinder of 2-mm radius and $2.5-\mathrm{km}$ length in a plasma with an electron density and temperature of $10^{12} \mathrm{~m}^{3}$ and $0.15 \mathrm{eV}$, respectively, the bias voltage required to collect $10 \mathrm{~A}$ is only $100 \mathrm{~V}$ ! But a tether is just a long, thin cylinder. Thus, if left uninsulated along part of its length, a tether can act as its own anode, capturing electrons efficiently over some positively biased segment.

For an orbiting, current-carrying tether, the bias will actually vary along the tether because of both the motional electric field and the ohmic voltage drop. The electron current to the tether will thus vary with height. Along the uninsulated part of the tether, the tether current will decrease with decreasing altitude, until the point is reached at which the tether is at zero bias with respect to the plasma (or the end of the tether is reached). Assuming there is a point of zero bias on the tether, then below that point an ion current (much smaller because of the high ratio of ion mass to electron mass) that decreases somewhat the average tether current will be collected, due to the negative bias.

The bias required to collect a given OML current varies as the inverse square of the collecting area, making it possible to reduce the required bias substantially by modestly increasing the collecting area. Since the current collected by an electron-collecting length $\mathrm{L}_{B}$ grows roughly as $\left(\mathrm{L}_{\mathrm{B}}\right)^{3 / 2}$, the tether can automatically accommodate drops in density by increasing the length of the collecting segment, shifting the zero bias-point downward (Figs. 2 and 3). This ability to maintain thrust levels with low electron densities makes nighttime reboost possible.

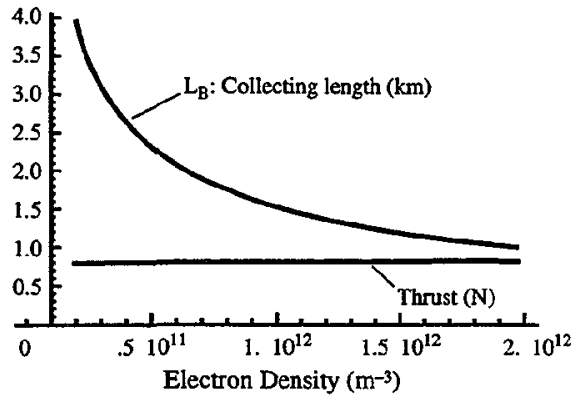

Fig. 3. Variation in thrust with electron density for a $10-\mathrm{km}$ tether with a $5-\mathrm{km}-10 \mathrm{ng}$ bare segment. Thrust drops only 10 percent as density drops by a factor of 10 . The reason is clear: the collecting length has increased from 1 to $4 \mathrm{~km}$. E.m.f. is $1,200 \mathrm{~V}$; input power $10 \mathrm{~kW}$.
Another important fact is that the OML current is identical for all cylinders with convex cross-sections of equal perimeter. ${ }^{3}$ With maximum crosswise dimension (here about $10 \mathrm{~mm}$ ) fixed by OML considerations, one is allowed to choose the cross-sectional shape. This frees us to choose a tape or ribbon-like tether, for example. The tape-like geometry gives somewhat better performance than a circular cross-section tether of equal length and mass and appears to have advantages related to deployment and thermal concerns. Tether geometry is one of the issues we will examine as part of the definition and design effort.

On the whole, the simplicity of the design, in addition to the ability to collect high currents and to accommodate density fluctuations by varying the collecting area, makes the bare-tether concept particularly attractive. Bare tethers are mostly free of the gross performance uncertainties that cloud the use of active, or sphere-like passive, contactors. The OML theory has been substantiated for both quiescent and flowing plasmas in the laboratory, and also in rocket and satellite flights, at moderate voltages. ${ }^{4-6}$

Ground simulation of electron collection in orbital conditions is possible because there is no need to reproduce the cylinder length-to-radius ratio in the lab; also, the orbital velocity should have no effect at the low radius-to-gyroradius ratio of interest. There are as yet no experimental results on tape-like collectors, or on the validity limits for OML theory at high voltages, but a series of plasma chamber tests are planned for the summer of 1996 . The 2-D geometry also makes a largescale program of particle-in-cell simulations feasible, and we anticipate using such simulations to study various tether geometries in our search for increased performance with lower mass. The proposed flight demonstration will serve to validate the performance of the anode system in space as a precursor to the ISS prototype.

Developing an ISS Reboost System. Our preliminary design for an electrodynamic tether thruster capable of delivering 0.5 to $0.8 \mathrm{~N}$ of thrust to the $I S S$ at a cost of 5 to $10 \mathrm{~kW}$ of electrical power consists of an 10-km-long aluminum tether in the form of a thick ribbon $(0.6 \mathrm{~mm}$ by $10 \mathrm{~mm})$. Despite its length, the tether would weigh only around $200 \mathrm{~kg}$. Since the bare portion of the tether is to act as our electron collector, a downward deployment of the tether is dictated by the physics of the eastward-moving platform.

The upper part of the tether will be insulated. There are two reasons for this. First, there is the necessity for preventing electrical contact from developing across the plasma between the upper portion of the tether and the Space Station, which (when the system is operating) are separated by an electrical potential difference of around a 
kilovolt. Beyond that, the insulation provides for greater thrust at a given input power. This comes from the fact that the largest tether-to-plasma bias occurs at the upper end, and decreases down the tether. A completely bare tether would draw the maximum current through the power supply, but the current would be strongly peaked at the upper end of the tether. Keeping the input power constant, we can substantially increase the average current in the tether, and hence the thrust, by insulating the tether over much of its upper portion, collecting current with the lower portion, and having a constant current in the upper part.

Determining the optimal fraction to insulate is part of the design effort for a "bare" tether reboost system. Our preliminary design has the upper 50 percent of the tether insulated. Even greater thrust during daytime operation could be obtained with a higher fraction, but the night-time adjustability would suffer.

The system provides flexibility, in the sense that the thrust obtained depends almost linearly on the input power, as seen in Fig. 4.

The bare-tether design has essentially "cured" the problem of day/night thrust fluctuations. But fluctuations in thrust due to fluctuations in the induced e.m.f. as the system encounters a varying geomagnetic field around the orbit are a fact of life for any tetherbased system. Figures 5 and 6 show the thrust variations around the ISS orbit with different input power levels.

Given the level of current the system may draw, the system will almost certainly require its own cathodic plasma contactor at the Station end. The contactors currently under development at NASA Lewis Research Center should be well suited for this function. If thrusts over $0.5 \mathrm{~N}$ are desired, it is likely that the system will also have to rely on the ISS's plasma contactor as well, or on a second dedicated contactor, since currents over the $10 \mathrm{~A}$ rating of the contactors could be required.

Before an operational electrodynamic tether reboost system for the ISS can be designed, a series of ground and space-borne experiments and computer simulations must be performed. In addition, thorough systems analyses must be performed to determine the physical integration and operational issues associated with its implementation on the ISS.

Among the issues to be addressed in the analyses of the reboost system are the attachment location for the tether, need for retrieval capability, microgravity impact, power interfacing, and safety. These are in addition to design issues specific to the tether itself, such as tether material, length, and geometry.

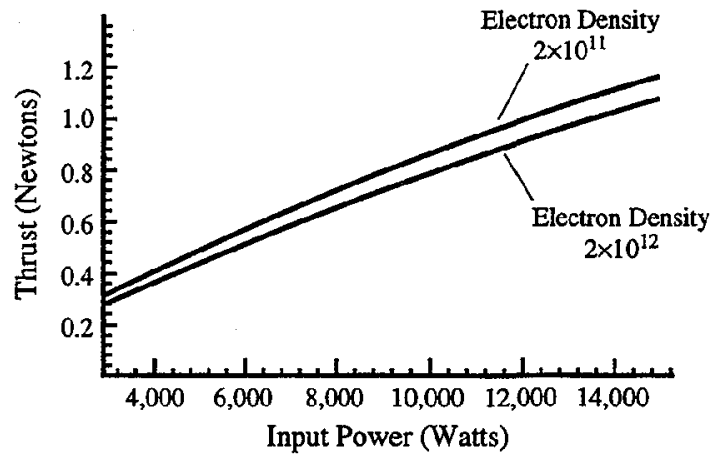

Fig. 4. Variation of thrust with input power for nominal $10-\mathrm{km}$ system. Motional e.m.f.: $1.2 \mathrm{kV}$.

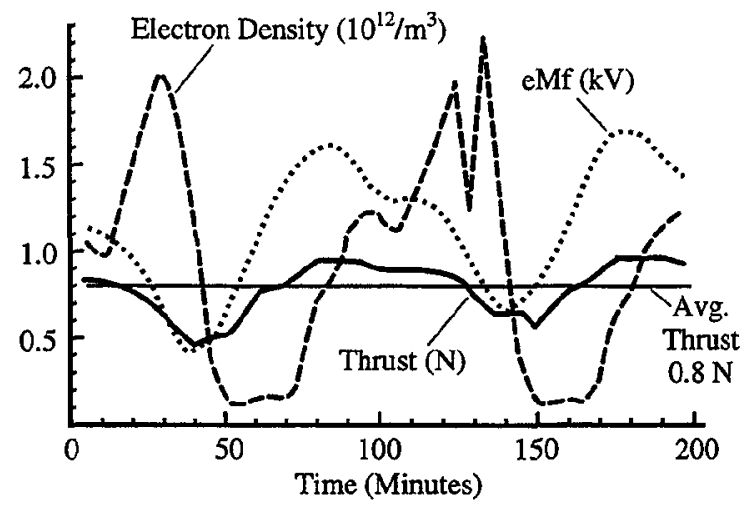

Fig. 5. Variations in thrust around two typical revolutions of the ISS orbit for the 10 . km-long electrodynamic tether thruster described in this section, as it operates at a constant power of $10 \mathrm{~kW}$. Dependence on electron density is weak, as expected. Thrust curve basically tracks e.m.t.

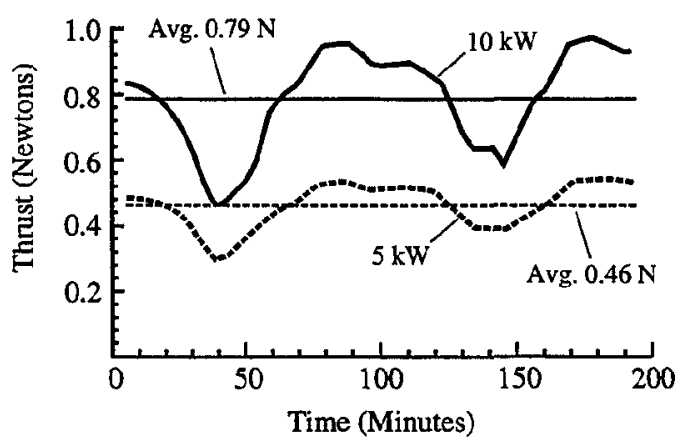

Fig. 6. Comparison of thrust generated for input powers of $5 \mathrm{~kW}$ and $10 \mathrm{~kW}$ for same tether and orbit as Fig. 5. 


\section{Assessment of Space Application and Benefits to the ISS}

Mission Benefit. The value in an electrodynamic tether reboost system lies in its ability to couple power generation with thrust. Heretofore, the electrical and propulsion systems have been effectively totally separate entities. Outfitting ISS with an electrodynamic reboost tether severs the most critical and constraining dependency on Earth-propellant resupply. The station can supply its own power but not its own propellant. Without an electrodynamic tether, the specter of Skylab and the words "reentry" and "atmospheric burnup" will forever haunt the minds of anyone who has an interest in the program. Add a tether and some additional storage capacity for supplies, and suddenly a 1-year interval between visits to the station becomes conceivable.

Even if the current frequency of resupply flights to the station is maintained, with an electrodynamic tether the station program has the option to trade kilowatts for increased payload capacity. Resupply vehicles can deliver useful cargo like payloads, replacement parts, and crew supplies rather than propellant. Within the range of 5 to $10 \mathrm{~kW}$, a crude approximation of $1,000 \mathrm{~kg}$ of user payload gained per $\mathrm{kW}$ expended per year appears reasonable; further analysis will refine this estimate.

As a bonus, propellantless reboost is exhaustless reboost: external contamination around the station is considerably reduced. The station reboost propellant is hydrazine. Any consumption of propellant may result in residual chemical deposits and contamination on the station's exterior surface. An electrodynamic tether provides a means to reboost the station without the complications of chemical combustion. The purity of the external environment for science payloads is enhanced, and beneficial operational impacts of reduced propellant exhaust on external systems and optics will be realized. Electrodynamic thrust truly represents solar power at its finest.

Yet another dimension to propellantless reboost must be considered. Station users have been allocated a minimum of 180 days of microgravity per year. Current planning essentially halts science activity during reboost maneuvers. Low-thrust electrodynamic tether reboost could be performed over long duration, as opposed to short duration, high-thrust propulsive maneuvers. The $0.5-$ to $0.8-\mathrm{N}$ thrust provided by a $10-$ $\mathrm{km}$ tether more than counteracts the station's atmospheric drag on a daily basis. Thus, the question arises, can an electrodynamic tether compensate for the drag while it is occurring, without disrupting the microgravity environment? Fluctuations in the induced voltages from the Earth's magnetic field and in electron densities will create "turbulence" through which the electrodynamic tether driven station must fly; can loadleveling control systems compensate for these pockets and maintain microgravity levels? In this case, a new realm of possibilities opens up for long-duration microgravity experiments. The allure of this selfpropelled space facility is certainly remarkable, and offers potential advantages.

Risk Reduction. Aside from replacement of failed components, an electrodynamic reboost tether on the station makes the vehicle itself essentially independent of propellant resupply from Earth. The primary resupply consideration becomes the inhabitants of the station and not the station itself. This is a new view for development of space operations. There ceases to be concern over the "180-day countdown to reentry at 150 nautical miles," which currently permeates every aspect of station mission planning. With the multibillion dollar investment in the vehicle virtually secured and free from concern over long resupply vehicle launch delays, particularly Russian Progress or FGB tanker delays, the program will be able to focus much more strongly on the ISS mission rather than on ISS itself.

Cost Pay Back. The cost of the proposed system comes in the form of the development, launch, and installation of an operational tether reboost system on the station. The payback comes in the form of reduced propellant upmass requirement. For 2003 to 2012, nearly $90,000 \mathrm{~kg}$ of propellant must be launched. Using a figure of $\$ 20,000$ per $\mathrm{kg}$, this represents a sum of $\$ 1.8$ billion. An electrodynamic tether supplying 90 percent of this requirement would reduce the operational cost by $\$ 1.6$ billion, paying for itself many times over. More modest estimates still result in a return on investment tens of times the cost of development and operation of an electrodynamic reboost tether.

It should also be noted that the commercial satellite industry may also be a beneficiary in the application of an electrodynamic reboost tether system. One of the most significant costs for satellites is launch weight, and a substantial portion of launch weight is propellant weight. If conventional propellant systems can be replaced by electrodynamic tether systems, new barriers will be broken in satellite longevity. It is repeatedly stated that satellite components are reliable but the available onboard propellant limits the useful life of the satellite. Initiating use of an electrodynamic tether at ISS may revolutionize design of future spacecraft.

Schedule Compatibility. If the Russians meet their current propellant resupply commitments, assembly phase priorities for power will most likely not change. Upon installation of the final photovoltaic arrays, there would presumably be sufficient power availability margin that the user community would be willing to 
trade 6 to $9 \mathrm{~kW}$ for an increase of 6,000 to $9,000 \mathrm{~kg}$ per year in payload upmass. Thus, the installation of an operational electrodynamic tether would have to be complete by 2002 or 2003 .

If, however, propellant resupply turns out to be the program's main bottleneck during assembly, work could be accelerated to install the system as early as late 1999 or early 2000 . Power margins are particularly thin during the initial build-up, so electrodynamic reboost would most likely come at the expense of payload user power.

\section{Conclusion}

Electrodynamic tether propulsion has many advantages over conventional propulsion systems for reboost of space systems such as the International Space Station and upper stages. The continued development of this technology has the potential to dramatically reduce to the life cycle costs of the ISS and provide a lowmass, low-consumable upper stage for in-space payload transfer.

\section{Acknowledgments}

The authors would like to express their gratitude to the team that helped assemble the proposal in response to the International Space Station Advanced Engineering Technology Development NASA Research Announcement (upon which this paper is based): Melody Herrmann (MSFC), Kai Hwang (Computer Sciences Corporation), Lee Marshall (Lockheed Martin Astronautics), and John Raitt (University of Utah).

\section{References}

1. Sanmartín et al., J. Propulsion \& Power, vol. 9, p. 353, 1993.

2. Laframboise, University of Toronto Institute for Space Research Report No. 100, 1966.

3. Laframboise and Parker, Phys. Fluids, vol. 16, p. $629,1973$.

4. Chung et al., Probes in Stationary and Flowing Plasmas, Springer, New York (1975).

5. Mercure, University of Toronto Institute for Aerospace Studies Report No. 202, 1976.

6. Szuszczewicz and Takacs, Phys. Fluids vol. 22, p. 2424, 1979. 